\title{
The Investigation of Mutual Interference Vortex Flow around Two Circular Cylinders by Flow Visualization and Pressure Measurement
}

\author{
Yoshifumi Yokoi ${ }^{1, a}$ and Rut Vitkovičová ${ }^{2}$ \\ ${ }^{1}$ National Defense Academy of Japan, Department of Mechanical Engineering, 1-10-20 Hashirimizu, Yokosuka 239-8686, Japan \\ ${ }^{2}$ CTU in Prague, FME, Department of Fluid Dynamics and Thermodynamics, Technická 4, 166607 Prague, Czech Republic
}

\begin{abstract}
In order to understand the aspect of the mutual interference flow from two circular cylinders, the visual observation experiment and the pressure measurement experiment were performed by use a water flow apparatus. Two circular cylinders with a diameter of $D=10 \mathrm{~mm}$ were used, and they have been arranged at staggered or tandem. The flow velocity was $U=0.25 \mathrm{~m} / \mathrm{s}(R e=U D / v, v$ is kinematic viscosity of fluid). The dye oozing streak method was used in the visualization experiment. In the pressure measurement experiment, the pressure on the surface of a circular cylinder was detected by the single tube manometer, and measurement was performed by image processing using a computer. As a result, distribution of the circular cylinder surface pressure coefficient $C_{P}$ corresponding to the flow pattern and it in each circular cylinder arrangement was obtained. The drag coefficient $C_{D}$ was calculated from the pressure coefficient $C_{P}$, and change of the resistance in each arrangement was found.
\end{abstract}

\section{Introduction}

In order to understand a "characteristics of flow", it is important to get to know a flow pattern and pressure. As general, "flow visualization" is performed for obtaining the flow pattern, and "pressure measurement" is performed using a pressure gauge for getting to know the pressure. "Flow visualization" is the technique of the ability to overlook the flow pattern, and it is a useful tool. In many case, the flow visualization is performed in comparatively low flow velocity. On the other hand, generally a manometer is used by the flow velocity from which enough water-head for measurement is obtained. So, in the low flow velocity performed by the "visualization", the pressure measurement by the manometer is very difficult. Therefore, "flow visualization" and "pressure measurement" have been performed by different flow velocity. This thing lacks reliability. An apparatus and a technique measurable with sufficient accuracy also by the low flow velocity performed by the "visualization" were required in pressure measurement. Yokoi [1] showed the proposal of a pressure measurement system which used the single tube manometer in the low flow velocity in response to such a demand. The pressure distribution on the surface of a single circular cylinder was measured using the proposed technique, and the good measurement result was obtained. As the study relevant to the interference problem of two bodies, Zdravkovich [2] introduces the studies that solve the drag and lift in the tandem arrangement case and parallel arrangement case and influence of two identical cylinders on the frequency of vortex shedding. Strykowski and Sreenivasan [3] deal experimentally and numerically the influence of the small cylinder (rear cylinder) and the formation of the vortex street behind the main cylinder at low Reynolds numbers. In their work indicate that there are positions of secondary small cylinder (rear cylinder) in which it can control the vortex street. Choi et al. [4] classify methods of flow control for the bluff cylinder and present results of comparing regions of attenuating instabilities using a small secondary cylinder. Mittal and Raghuvanshi [5] solve the influence of a small secondary cylinder on the main cylinder (primary cylinder) using numerical simulations. They compare with their results and previous experimental results and focus on the causes of the influence of the control cylinders on the main cylinder. Yokoi and Hirao [6-10] is reporting the result of the study shown by the streak line about the vortex shedding from the circular cylinder which is oscillating in the direction of flow using the flow visualization technique based on a pouring streak method. Vitkovičová et al. [11, 12] is reporting the result of study which showed the interference flow from two circular cylinders arranged staggered using the flow visualization technique based on a suspension method by the particle pass line. Then, the visualization experiment by the streak line method was performed on the same conditions as a previous experiment for the database creation in comparison with the previous experimental result [11]. Although there is much study which has described the structure of the flow, the study which has presented the relationship of the surface pressure and the flow is not seen. In order to understand more deeply the structure of the flow in which

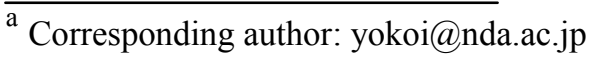


it interfere each other, the study which measures surface pressure is required.

In this study, the flow visualization experiment and the pressure measurement experiment on the surface of a circular cylinder were performed by the same flow velocity using the closed circuit water channel. The tracer ink of a different color using a pouring streak method was made to ooze from each circular cylinder in the flow visualization experiment. In the pressure measurement experiment, the image processing technique which used a computer and drawing software was used. As a result, the aspect of vortex shedding from each circular cylinder and the aspect of mutual interference flow were shown vividly. Surface pressure distribution of each circular cylinder by circular cylinder arrangement was found.

\section{Experimental apparatus and method}

\subsection{Outline of experimental apparatus}

The experimental apparatus consists of a small closed circuit water channel, visualization equipment, a pressure measuring device, and image record equipment. The closed circuit water channel is a vertical circulation type of $2.5 \mathrm{~m}$ in length, $0.3 \mathrm{~m}$ in width, and $1 \mathrm{~m}$ in height, and the volume of water is 0.4 tons. The water channel consisted of water flow generation equipment ( 1 axial flow type pump), rectification device, test section and 4 corner parts with guide vanes. The test section is $1.2 \mathrm{~m}$ in length, $0.3 \mathrm{~m}$ in width, $0.2 \mathrm{~m}$ in depth, and the flume structure with the surface of the water. The test section is a product made from transparent acrylics. The maximum flow velocity in the test section was $1.3 \mathrm{~m} / \mathrm{s}$. At the time of the experiment, the water level was set as $0.2 \mathrm{~m}$. The flow is generated by carrying out input operation of the operation frequency $f \mathrm{~Hz}$ in a digital inverter. The flow velocity $\mathrm{v}$ is obtained by $U=0.0327 \times f(\mathrm{~Hz})$. The board for circular cylinder attachment is prepared in the open section of the test section. The line which shows a direction is drawn on the board. It is the mechanism in which direction of a pressure hole can be set by piling up the line and mark of a circular cylinder end. The photograph of closed circuit water channel experimental apparatus is shown in Fig. 1.

The visualization apparatus consists of two circular cylinders for visualization, a tracer ink feed unit and a lighting installation. The diameter $(D)$ and the length of test circular cylinder are $10 \mathrm{~mm}$ and $300 \mathrm{~mm}$, respectively. The screw for circular cylinder fixation (M4) is prepared in the circular cylinder end, and the tracer ink introduction way is provided in the inside of the circular cylinder from the section of the screw to the tracer oozing port on the surface of circular cylinder. Two tracer ports for visualization were provided in the position of \pm 60 degrees from the cylinder stagnation point. They are located near $100 \mathrm{~mm}$ from the circular cylinder other end. When installing the circular cylinder in the water tank test section, the tracer oozing position is $100 \mathrm{~mm}$ under the water surface. For convenience, front side and rear side cylinders are called the "1st cylinder" and "2nd cylinder", respectively. In order to reduce mixture by

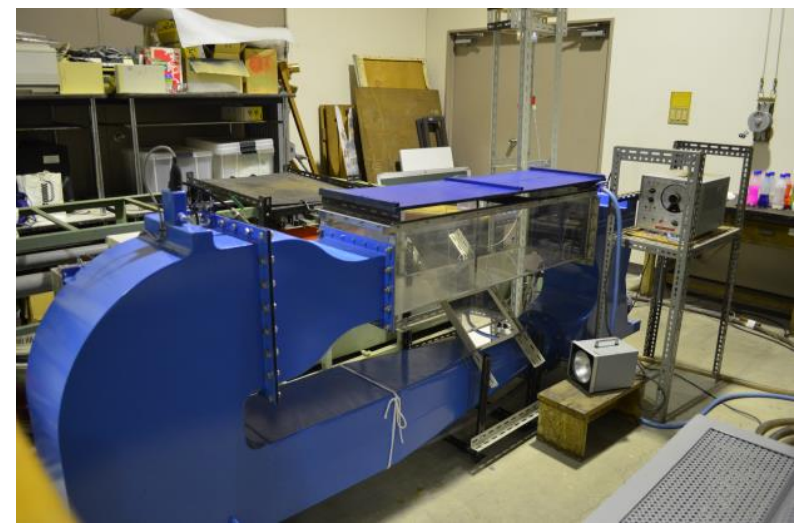

Figure 1. Aspect of experimental apparatus.

tracer oozing, the depth positions of the oozing port differ in the 1 st cylinder and the 2 nd cylinder. The oozing port of 1 st cylinder is provided in the water surface side, and the oozing port of 2 nd cylinder is provided in the bottom side. The gap between both is $2 \mathrm{~mm}$. The ink tank is installed above the water surface, and a set of the clamps and opening-and-closing clips for flow regulation are formed in the vinyl tube which connects the circular cylinder to the ink tank. As for the lighting installation, a halogen light of $500 \mathrm{~W}$ was used.

The pressure measuring device consists of a circular cylinder for measurement and a single tube manometer with sale. The cylinder diameter $(D)$ is $10 \mathrm{~mm}$, and the cylinder length is $300 \mathrm{~mm}$. The screw for setting is prepared in the upper edge of the circular cylinder, and the marker in which a direction is shown is describing in the edge. The small hole which is the diameter of $1 \mathrm{~mm}$ for pressure measurement is provided in the position which is $100 \mathrm{~mm}$ from the circular cylinder lower end. It connects with the single tube manometer lower end by a vinyl tube through the taxiing way provided in the circular cylinder from the small hole. The manometer main part is glass and uses a little thick tube (the diameter of inner is $10 \mathrm{~mm}$ ) in consideration of the surface tension of water. The scale made from the steel for water level reading is attached in the side of the glass tube.

The video recording apparatus consists of a camera for photography, and a personal computer for image processing. The camera for photography used the digital camera (Nikon D40) and the digital camcorder (WAT250D2) properly by the use. Those cameras fixed to the tripod mount were set in the manometer front. The personal computer (NEC LaVie) commercial note type was used for image-processing measurement. Measurement of the length of picture was performed using "Microsoft Paint" [13] installed as application software. The video signal converter (Alterplus AX-200) was used for dealing with the image signal from a digital camcorder with a personal computer.

\subsection{Flow visualization}

The flow visualization technique used in this experiment is a pouring streak method. Tracer ink is led to the inside of the circular cylinder using a vinyl tube from a tracer 
tank, and it carries out by adjusting the valve travel of the clamp which provided that the tracer ink of the grade which does not destroy a boundary layer from the oozing port on the circular cylinder surface oozed in the middle of the vinyl tube. The used tracer ink is the poster color thinned moderately. "Pink" and "turquoise blue" were used with the 1st cylinder and the 2nd cylinder, respectively.

\subsection{Pressure measurement}

A pressure measurement cylinder is set in a test section. The pressure tube from the circular cylinder is connected to the single tube manometer. The focus and zoom of the photography camera are adjusted. Those conditions are held after completing this adjustment until a series of photography is completed. The water level of the manometer in a stream state of rest is photographed. The stream is generated. The water-head of the manometer in each direction angle of the circular cylinder is photographed and recorded. The obtained picture is downloaded to the computer, the image-processing measurement is performed, and a pressure coefficient $C_{P}$ is calculated. The photographed image data is introduced into the computer. When the digital camera was used, the work was performed through memory card (SD card). When the digital camcorder was used, the work was performed through the USB conversion unit.

The "Paint" is started and the target picture file is opened. A reference position is decided in the displayed picture. Here, a reference position uses the same position over a series of measurement. A square is chosen from the "figure" in the section of a menu display of the software screen upper part. The displayed square is drawn on the manometer and scale. It meets with the reference position which determined one of the sides of the square. Another side is met with the manometer water-head position. For example, the upper side will be met with the manometer water-head position if the base of the square is met with the reference position. Since the information on the length of two sides of the square (the number of horizontal pixels, $x$, the number of vertical pixels) is displayed on the software screen bottom, the information on the number of vertical pixels is read. The actual dimension per pixel is obtained by authorization. For example, it is investigated by the box function whether an actual length of $10 \mathrm{~mm}$ shown on the screen is displayed with how much number of pixels. The thickness of the drawing line sets up so that it may be easy to operate it. A reference position is determined as the scale shown on the screen.

It is set as 0 degree of directions, and the length between the water level and the reference position at the time of stationary is measured. The length is called $l_{0}$. A stream is generated and the length between the manometer water-head for every setting angle and the reference position is measured. The length is called $l_{\theta}$. The pressure coefficient $C_{P}$ is calculated by $\left(l_{\theta}-l_{0}\right) /\left(\rho v^{2} / 2\right)$. In the case of the 2 nd circular cylinder, the position (direction) which dynamic pressure requires with a setting position changes with influences of the 1 st
Table1. Setting positions of 2nd cylinder.

\begin{tabular}{|c|c|}
\hline & $(x / D, y / D)$ \\
\hline Posi1 & $(4.30,-1.20)$ \\
\hline Posi2 & $(3.20,-0.80)$ \\
\hline Posi3 & $(2.40,-0.80)$ \\
\hline Posi4 & $(1.95,-0.80)$ \\
\hline Posi5 & $(2.00,-0.60)$ \\
\hline Posi6 & $(1.50,-0.35)$ \\
\hline Posi7 & $(1.50,0.00)$ \\
\hline
\end{tabular}

circular cylinder. So, only the 2 nd circular cylinder is set as the specified position, and it determines for the manometer water level $l_{0}$ at the time of stationary. Next, it determines for the manometer water-head $l s_{0}$ when

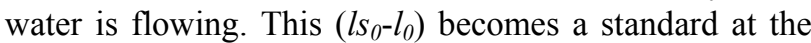
time of determining for a pressure coefficient $C_{P}$. Then, the 1 st circular cylinder is set. Here, at least all directions rotate the 2nd circular cylinder for every angle, and manometer water-head $l_{\theta}$ is measured. A pressure coefficient $C_{P}$ is required by $\left(l_{\theta^{-}} l_{0}\right) /\left(l_{s_{0}}-l_{0}\right)$, and the distribution is obtained.

\subsection{Experimental parameters}

The main experimental parameters are the flow velocity, the arrangement position of two cylinders and the direction of pressure measurement. The flow velocity was performed by $U=0.25 \mathrm{~m} / \mathrm{s}$. The Reynolds number based on this flow velocity and the circular cylinder diameter was 2500 . And also when the flow velocity is $0.25 \mathrm{~m} / \mathrm{s}$, according to Bernouilli's theorem, an anticipation waterhead is $3.2 \mathrm{~mm}$. Arrangement of two circular cylinders is seven kinds. The 1 st cylinder position was put on origin $(\mathrm{x}, \mathrm{y})=(0,0)$, and the position of the 2 nd cylinder was changed as shown in Table 1. An angle of direction is every 10 degrees in the range of 0 to 360 degrees.

\section{Experimental results and discussion}

\subsection{Visualized flow pattern of two cylinders}

It is one of the most interesting things of this study to conduct visualization investigation of an aspect that install another circular cylinder behind cylinder and the vortex flow is controlled. It was observed that the aspect of the flow of the 2nd cylinder completely differs from the aspect of the flow when the 2 nd cylinder is placed independently through all experimental conditions. In staggered arrangement like this study, flow pattern can divide roughly into the following three types by the behaviour of the separating shear layer of the 1st cylinder by the side of the 2 nd cylinder. (1) The state completely rolled by the separating shear layer before the 2nd cylinder. (2) The state of colliding with the 2 nd cylinder before having been rolled by the separating shear layer since the 2 nd cylinder is located in the outside of the separating shear layer. (3) The state where the flow from the 2 nd cylinder is taken into the re-circulation flow of 


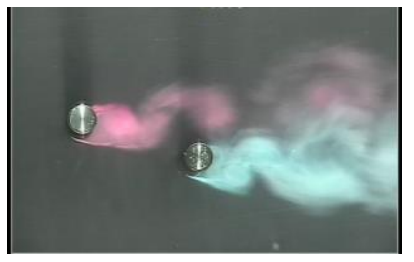

(a)

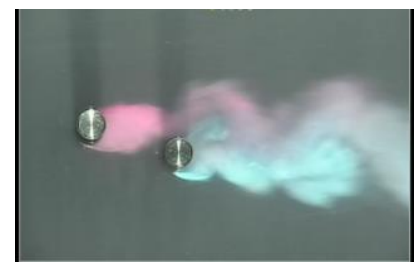

(b)

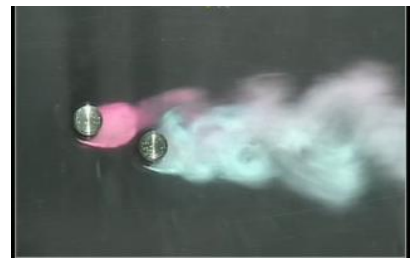

(c)

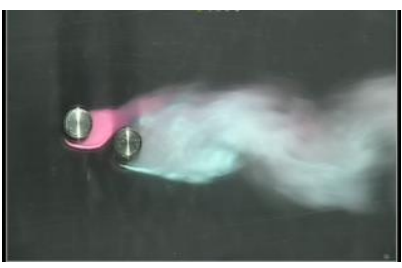

(d)

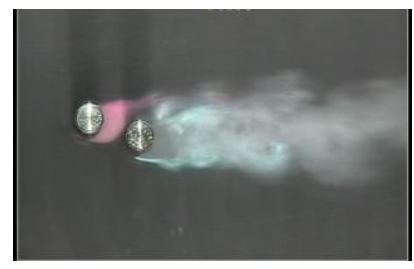

(e)

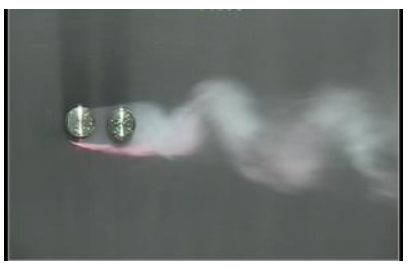

(f)

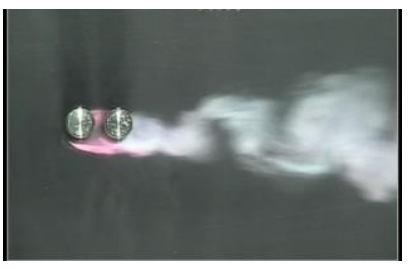

(g)

Figure 2. Aspect of the flow when varying the position of 2nd cylinder, $R e=2500$; (a) Posi1, (b) Posi2, (c) Posi3, (d) Posi4, (e) Posi5, (f) Posi6, (g) Posi7.

the 1st cylinder since the 2nd cylinder is located inside the separating shear layer.

Figure 2 shows the aspect of the flow when varying the position of the 2nd cylinder. In "Posi1" case, the vortex is alternately discharged from the 1st cylinder, and the vortex by the side of the 2nd cylinder has collided with the 2nd cylinder. It has united with the vortex street which could draw near to the 1st cylinder side the vortex shedding from the 2nd cylinder under the influence, and was discharged from the 1st cylinder. In "Posi2" case, before having been rolled by the separating shear layer discharged from the 1st cylinder, it has collided with the 2nd cylinder. Therefore, the Karman vortex was not produced from the 1st cylinder, but the vortex shedding from the 1st cylinder has assimilated to the vortex shedding of the 2nd cylinder. In "Posi3" case, the separating shear layer from the 1st cylinder is flowing into the re-circulation region behind the 1st cylinder. In "Posi4" case and "Posi5" case which the position of the 2nd cylinder approaches, the phenomenon seems to be more remarkable. In "Posi6" case or "Posi7" case, the 2nd cylinder is placed inside roll up of the separating shear layer discharged from 1st cylinder. Therefore, the separating shear layer from 2 nd cylinder is carried to the re-circulation flow of the 1st cylinder, and forms the stagnation region. The vortex shedding of two circular cylinders forms the Karman vortex just like the vortex shedding from one compound body.

\subsection{Pressure distribution on surface of cylinder}

It is interesting important in this research to obtain the pressure distribution result in low Reynolds number. The surface pressure distribution of each circular cylinder corresponding to three typical flow pattern classified by the influence of boundary layer separation from the 1st circular cylinder is shown in Fig. 3. In the figure, an abscissa is a cylindrical angle and an ordinate is a pressure coefficient. Here, the pressure coefficient is normalized with the value of an angle of 0 degrees of a single circular cylinder. The black dot mark shows the case of a single circular cylinder, and, as for the white circle mark, the 1st circular cylinder and a double white circle mark show the 2nd circular cylinder. An attention point is in the region behind the cylinder. In the case of Posi1, although pressure distribution of the 2nd circular cylinder showed the same pressure recovery as the case of a single circular cylinder, enough pressure recovery was not seen in pressure distribution of the 1 st circular cylinder. The value of the pressure coefficient of the 2 nd circular cylinder has larger relationship than the value of the pressure coefficient of the 1st circular cylinder. In the case of Posi2, pressure distribution of the 1st circular cylinder showed the same pressure recovery as the case of a single circular cylinder. In connection with it, pressure distribution of the 2nd circular cylinder showed pressure recovery further. Also at this time, the value of the pressure coefficient of the 2nd circular cylinder has larger relationship than the value of the pressure coefficient of the 1st circular cylinder. Circular cylinder arrangement of Posi1 and Posi2 is in the state completely rolled before the 2 nd circular cylinder by the separating shear layer from the 1st circular cylinder. When the 2nd circular cylinder approached the 1st circular cylinder in such the state, it was expected that both of pressure distribution was shifted towards pressure recovery, with mutual relationship maintained. In the case of Posi3, even if it compares the case of the 1st circular cylinder, and the case of the 2nd circular cylinder based on the case of a single circular cylinder, a difference is scarcely seen. In the relationship of the pressure coefficient, the value of the pressure coefficient of the 1st circular cylinder is larger than the case of a single circular cylinder, and the value of the pressure coefficient of the 2 nd circular cylinder is smaller than the case of a single circular cylinder. In the case of Posi4, pressure coefficient distribution of the 1st circular cylinder shows the same situation as the case of a single circular cylinder. There is 

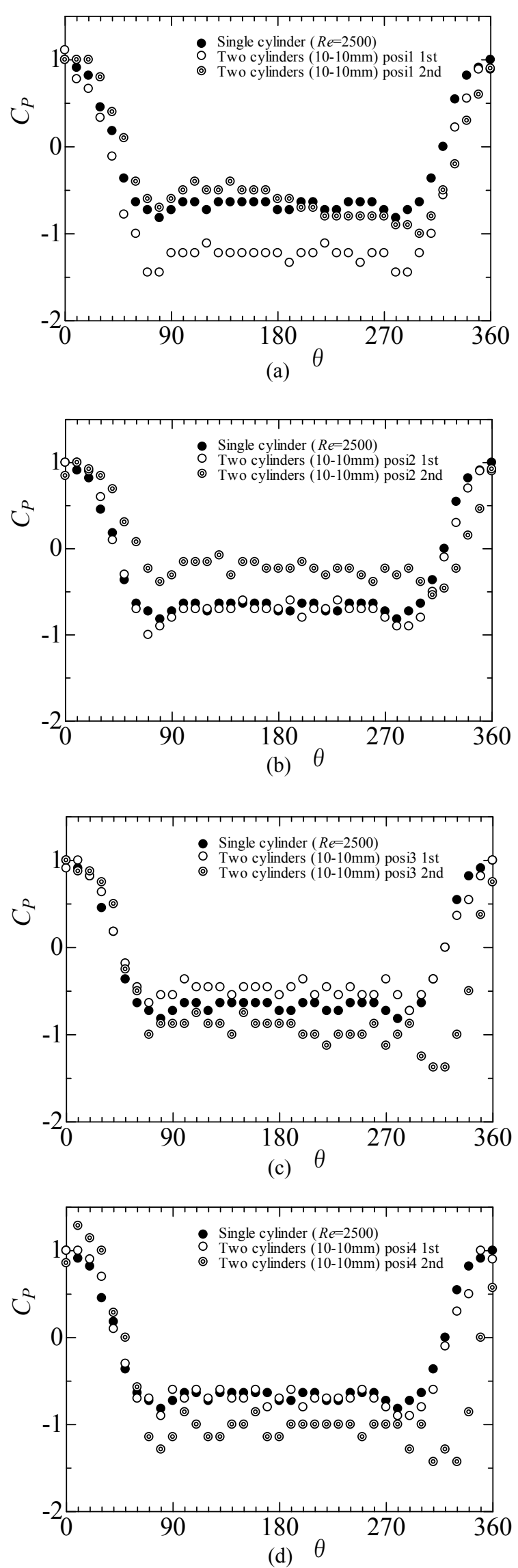
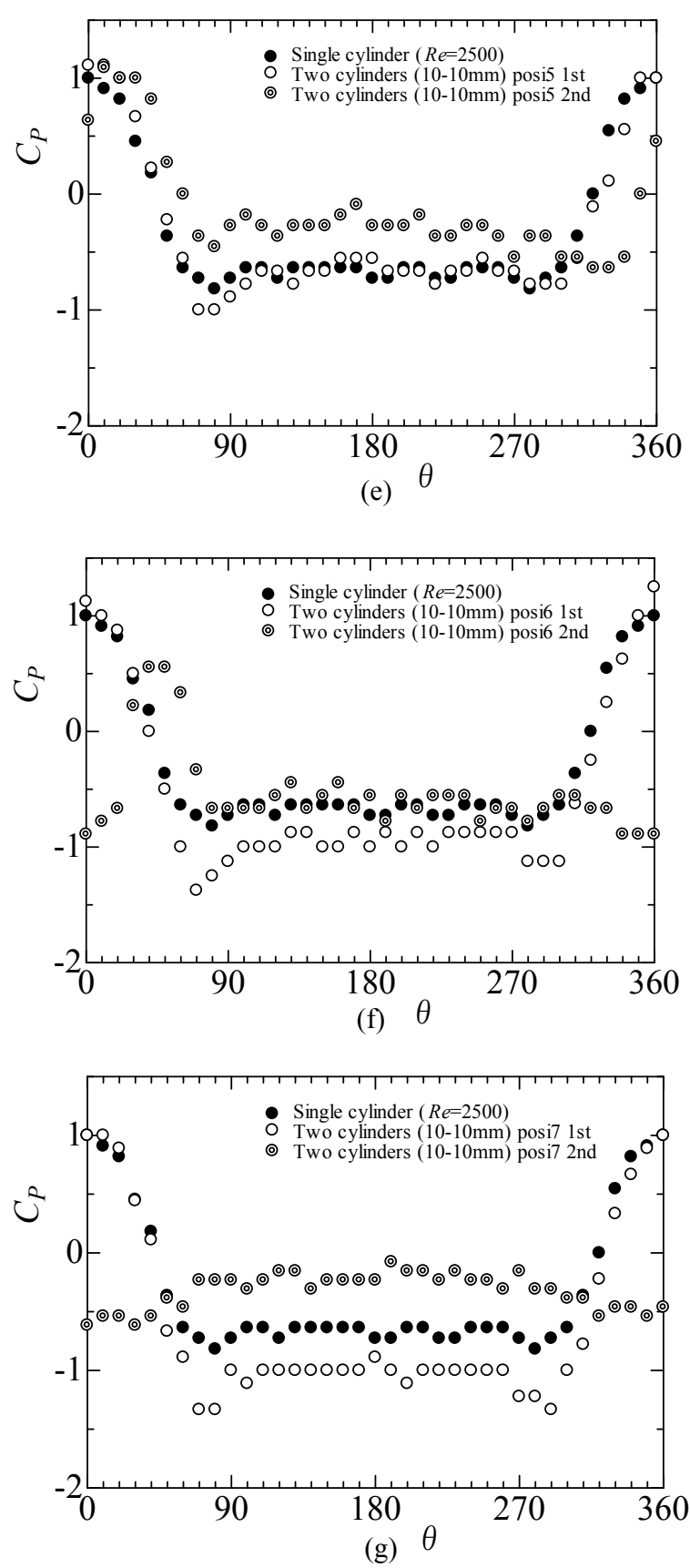

Figure 3. Surface pressure coefficient distribution of each circular cylinder for every circular cylinder arrangement in $R e=2500$, (a) Posi1, (b) Posi2, (c) Posi3, (d) Posi4, (e) Posi5, (f) Posi6, (g) Posi7. 
Table 2. Calculated drag coefficient and fluctuation ratio.

\begin{tabular}{|c|c|c|}
\hline Position & \multicolumn{2}{|c|}{$\begin{array}{r}C_{D} \text { Coefficient (Fluctuation } \\
\text { ratio \%) }\end{array}$} \\
\hline Single & \multicolumn{2}{|c|}{0.961} \\
\hline & 1st Cylinder & 2nd Cylinder \\
\hline Posi1 & $1.222(+27.16)$ & $0.747(-22.27)$ \\
\hline Posi2 & $0.927(-3.54)$ & $0.506(-47.35)$ \\
\hline Posi3 & $0.783(-18.52)$ & $0.793(-17.48)$ \\
\hline Posi4 & $0.950(-1.14)$ & $0.875(-8.95)$ \\
\hline Posi5 & $0.934(-2.81)$ & $0.403(-58.06)$ \\
\hline Posi6 & $1.095(+13.94)$ & $0.173(-82.00)$ \\
\hline Posi7 & $1.134(+18.00)$ & $-0.283(-129.45)$ \\
\hline
\end{tabular}

relationship that the value of the pressure coefficient of the 1 st circular cylinder is larger than the value of the pressure coefficient of the 2 nd circular cylinder. In the case of Posi5, pressure coefficient distribution of the 1st circular cylinder shows the same situation as the case of a single circular cylinder like the state of Posi4. However, it is shown that the value of the pressure coefficient of the 2nd circular cylinder is larger than the value of the pressure coefficient of the 1st circular cylinder. Arrangement of the 2nd circular cylinder of Posi3, Posi4, and Posi5 is in the state of blocking roll up of the separating shear layer from the 1 st circular cylinder. In the relationship of arrangement of the circular cylinder of Posi4 and Posi5, it is very interesting that such a large difference occurred only by a little differing. It is found that the spatial relationship of two circular cylinders of this state affects surface pressure greatly. In the case of Posi6, although pressure recovery of the 1st circular cylinder and the 2 nd circular cylinder is comparable, it can grasp that the profile of pressure distribution differs from the profile of pressure distribution of a single circular cylinder. It is shown that the value of the pressure coefficient of the 1 st circular cylinder is smaller than the value of the pressure coefficient of a single circular cylinder. In the case of Posi7, it is shown that the value of the pressure coefficient of the 1st circular cylinder is smaller than the value of the pressure coefficient of a single circular cylinder like the case of Posi6. And with the 2 nd circular cylinder, the value of a pressure coefficient shows the almost constant value. It can imagine that the 2 nd circular cylinder is completely taken into the wake of the 1 st circular cylinder.

\subsection{Variation of drag coefficient}

If it integrates with the direction component of a flow of the pressure coefficient $C_{P}$ on the surface of a circular cylinder, a drag coefficient $C_{D}$ can determine. Table 2 shows the value and fluctuation ratio of a drag coefficient

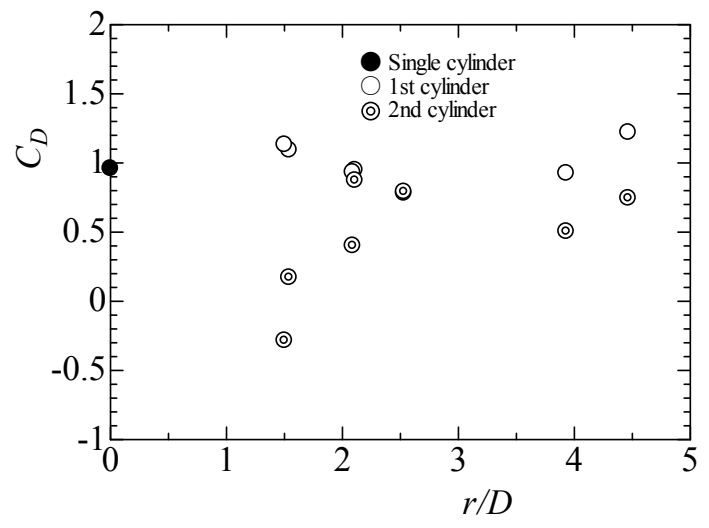

Figure 4. Variation of drag coefficient $\left(C_{D}\right)$ to distance between two circular cylinders $(r)$.

$C_{D}$ which were calculated by each circular cylinder arrangement. Figure 4 shows the variation of a drag coefficient to the distance between two circular cylinders. Here, the Simpson method was used in the integration. The fluctuation ratio is defined in the following. (Calculated drag coefficient - Drag coefficient of a single circular cylinder $) /($ drag coefficient of single circular cylinder) x100 (\%). The value of the drag coefficient of the single circular cylinder obtained by this measurement is 0.961 , and was well in agreement with the previous result [14]. This means that this measurement technique is appropriate. So, the value of the drag coefficient of two circular cylinders obtained by this measurement is considered to be what has high reliability.

The following matters can be read in Fig. 4. In the 2nd circular cylinder, the phenomenon of thrust acting and reducing the value of a drag coefficient is not dependent on an arrangement place, and occurs. On the other hand, in the 1st circular cylinder, it can be found that there is a change in the value of a drag coefficient depending on the arrangement position of the 2 nd circular cylinder.

\section{Conclusions}

The visualization experiment and the measurement experiment of circular cylinder surface pressure were performed by the same flow velocity using the closed circuit water channel. The following conclusions were obtained.

(1) The aspect of interference flow changes with the position of 2 nd cylinder.

(2) In three typical patterns with which the influence of boundary layer separation from the 1 st circular cylinder is distinguished, the measurement result of the surface pressure distribution of each two circular cylinders was obtained.

(3) If the presenting measurement technique is used also by a little water-heads which the low flow velocity produces, measurement of surface pressure distribution can be performed and the drag coefficient will be obtained. 
(4) Surface pressure distribution of each two circular cylinder in the state where arrangement differs was obtained, and the aspect of the difference in the pressure distribution by the state was found.

(5) The drag coefficient $C_{D}$ was calculated from the pressure coefficient $C_{P}$, and the variation of the drag coefficient in each arrangement was found.

\section{References}

1. Y. Yokoi, Proceedings of Experimental Fluid Mechanics, USB, 694(2018)

2. M. M. Zdravkovich, J. Fluids Eng. 99(4). 618633(1977)

3. P. J. Strykowski, K. R. Sreenivasan, J. Fluid Mech. 218, 71-107(1990)

4. H. Choi, I. Lee, W. P. Jeon, Annu. Rev. Fluid Mech. 40, 113-139(2008)
5. S. Mittal, A. Raghuvanshi, Int. J. Numer. Math. Fluids 35, 421-447(2001)

6. Y. Yokoi, K. Hirao, J. Fluid Science and Technology 4-2, 401-414(2009)

7. Y. Yokoi, K. Hirao, EPJ Web of Conf. 45, 01098(2013)

8. Y. Yokoi, K. Hirao, EPJ Web of Conf. 67, 02131(2014)

9. Y. Yokoi, K. Hirao, J. Fluid Science and Technology 9-3, 0057(2014)

10. Y. Yokoi, K. Hirao, EPJ Web of Conf. 92 02116(2015)

11. R. Vitkovičová, J. Nozicka, J. Cizek, V. Skala, EPJ Web of Conf. 92, 02110(2015)

12. R. Vitkovičová, Vladislav Skala, EPJ Web of Conf. 114, 02138(2016)

13. https://en.wikipedia.org/wiki/Microsoft_Paint

14. T. Toyokura, K. Kamemoto, Fluid $\overline{d y n a m i c s}$ (in Japanese), 233(1981) 